BULL. AUSTRAL. MATH. SOC.

$20 \mathrm{D} 10$

VOL. $33(1986), 75-80$.

\title{
SUB-DIRECT PRODUCT CLOSED FITTING CLASSES
}

\author{
R.A. BRYCE
}

It is shown that in the Fitting class of all finite $p-b y-q$

groups, where $p$ and $q$ are different primes, there is among the sub-direct product closed sub-Fitting classes a unique maximal one : it consists of the groups whose minimal normal subgroups are central.

Several investigations, for example $[1,2,3,5]$ consider the problem of classifying metanilpotent fitting classes closed under an additional closure operation. To date the least satisfactory results concern subdirect product closure. In contrast to subgroup closure, quotient group closure and saturation there are sub-direct product closed metanilpotent Fitting classes which are not primitive saturated formations: $\mathcal{C}$, the class of finite soluble groups whose minimal normal subgroups are all central, is a sub-dixect product closed Fitting class, so $S_{p} S_{q} \cap \mathrm{C}$ is a non-quotient group closed, and therefore non-primitive, Fitting class when $p, q$ are different primes. The purpose of this note is to prove the following result.

THEOREM. If $p, q$ are different primes then $S_{p} S_{q} \cap \mathcal{C}$ is the conique maximal sub-direct product closed Fitting class contained in $s_{p} s_{q}$.

Received 2 May 1985.

Copyright clearance Centre, Inc. Serial-fee code: 004-9727/86 $\$ A 2.00+0.00$. 
A proof of this result in the case $q \mid p-1$ is given in [3] and the present proof closely follows that given there. To prove the theorem it suffices to show that if $F$ is a sub-direct product closed Fitting class contained in $S_{p} S_{q}$, and if $F \notin \mathcal{C}$, then $F=S_{p} S_{q}$.

The following lemma will be used several times in the proof.

(1) Suppose that $A_{1}, A_{2}$ are operator groups for a nilpotent group $B$, and suppose that $x$ is a sub-direct product closed Fitting class. If $\left(A_{1} \times A_{2}\right) B \in X$ then $A_{1} B \in X$.

For, $\quad H=\left(A_{1} \times A_{1} \times A_{2}\right) B \in R_{0}\left\{\left(A_{1} \times A_{2}\right) B\right\} \subseteq X$. Since $H /\left(A_{1} \times 1 \times 1\right) \in X$ it follows from Lemma 1.1 of [2] that $H /\left(1 \times A_{1} \times A_{2}\right) \cong A_{1} B \in X$

With $F$ as above we show that

$$
D_{p}^{q} \in F
$$

For, since $F \notin C$ there is a group $G \in F$ with a non-central minimal normal subgroup $M . M$ is a $p$-group and $G / \mathbb{E}_{G}(M)$ is a $q$-group. Consider the natural splitting extension $R=M G$. There are two natural homomorphisms of this group onto $G$, one defined by $(g, m) \rightarrow g$, the other defined by $(g, m) \rightarrow g m$. Since their kernels intersect trivially it follows that $R \in F$. However, if

$$
C=\left\{(g, 1): g \in \mathbb{E}_{G}(M)\right\}
$$

then $C \exists R, C \cap M=1$ and $R / M C$ is nilpotent, so by Lemma 1.1 of [2], $R / C \in F$. It follows that $F$ contains a group $S$ with the following structure : $S=M D$ where $M$ is an elementary abelian $p$-subgroup, the unique minimal normal subgroup of $S$, and $D$ is a non-trivial q-group. Consequently if $Q$ is a subgroup of order $q$ contained in $\zeta_{1}(D)$ then $M Q \in S_{n} F=F ;$ and if $M_{1}$ is an irreducible component of $M_{Q}$ then $M=M_{1} \oplus M_{2}$ where $M_{2}$ admits $Q$. Hence by (1) $D_{p}^{q} \cong M_{1} Q \in F$.

Let $U(p, q)$ denote the class of all groups with a normal $p$-subgroup complemented by a subgroup of order $q$. The next result is an immediate corollary of $(2)$. 
(3) If $H \in U(p, q)$ and $\bar{o}_{p}(H)$ is elementary abe lian then $H \in F$.

(4) If $u(p, q) \subseteq F$ then $F=s_{p} s_{q}$.

Let $H=A B, A \exists H, A \cap B=1, A \in S_{p}, B \in S_{q}$. We show by induction on $|B|$ that $H \in F$. If $B$ is not cyclic then $B$ is a product of normal subgroups $B_{1}, B_{2}$ of smaller order than $|B|$ so by induction

$$
\begin{aligned}
A B & \in N_{0}\left\{A B_{1}, A B_{2}\right\} \\
& \subseteq F .
\end{aligned}
$$

If $B$ is cyclic, say of order $q^{\alpha}$ then the case $\alpha=1$ is covered by the hypothesis of (4); so suppose $\alpha>1$. In this case we may regard $B$ as a subgroup of the group $W=C_{q}$ wr $C_{q}^{\alpha-1}$. Form the twisted wreath product (see pp. 227-8 of [4])

$$
T=A \operatorname{twr}_{B} W \text {. }
$$

Then since $W$ is a product of subnormal subgroups of order less then $|B|$, $T \in F$. But then

$$
\left(\left.\mathrm{A}\right|^{\mathrm{W}}\right) \mathrm{B} \in \mathrm{S}_{\mathrm{n}} \mathrm{F}=\mathrm{F} \text {. }
$$

Since by Lemma 3.3 of [4]

$$
\left.A\right|^{W} \mid B \cong A \times K
$$

where $K$ admits $B$ we conclude from (1) that $A B \in F$ to complete the induction.

(5) Let $H \in U(p, q)$ and let $V$ be a $z_{\rho}$ H-module having a submodule $U$ with $V / U$ being completely reducible. If $p$ has order $d$ modulo $q$ then $(d V) H \in F$ if $U H \in F$ and $(d U) H \in F$ if $V H \in F$.

Write $r$ for a cyclic group of order $q$ and let $N$ be a faithful irreducible $Z_{p} r$-module. Since $V / U$ is completely reducible for $H$ we may write

$$
V \# N / U \# N=\underbrace{\infty}_{i=1} X_{i} / U \# N
$$

where each $X_{i} / U \# N$ is irreducible for $H \times \Gamma$. Then, $O_{p}(H)$ being in the kernel of $X_{i} / U \# N$, and faithfully and irreducible represented abelian 
groups being cyclic, $K_{i}$, the kernel of $X_{i} / U \# N$ in $H \times \Gamma$, satisfies $o_{p}(H) \leq K_{i}$ and $H \Gamma=K_{i} \Gamma$. It follows that if we write $y_{0}=U \# N$ and

$$
Y_{i}=\sum_{j=1}^{i} X_{j}, \quad 1 \leq i \leq r,
$$

then $\quad Y_{i} K_{i+1} \forall Y_{i+1} K_{i+1} \in N_{0}\left\{Y_{i} K_{i+1}, Y_{i+1}\right\}, \quad 0 \leq i \leq r-1$.

Consequently

(6) $y_{i} K_{i+1} \in F$ if and only if $y_{i+1} K_{i+1} \in F, \quad 0 \leq i \leq p-1$.

Also

$$
Y_{i} K_{i+1} \forall Y_{i}(H \times \Gamma) \in N_{0}\left\{Y_{i} K_{i+1}, Y_{i} \Gamma\right\}
$$

and

$$
Y_{i+1}{ }_{i+1} \forall Y_{i+1}(H \times \Gamma) \in N_{0}\left\{Y_{i+1} K_{i+1}, Y_{k+1} \Gamma\right\}
$$

Then, since $Y_{j} \Gamma \in F$ for all $j$ by (3), we have that if $Y_{i} H \in F$ then $y_{i} K_{i+1} \in F$ by (7) whence $y_{i+1} K_{i+1} \in F$ by (6) and then that $y_{i+1} H \in F$ by (8). A similar argument shows the converse and hence $y_{i} H \in F$ if and only if $Y_{i+1} H \in F \quad(O \leq i \leq n-1)$. By induction therefore $(U \# N) H \in F$ if and only if $(V \# N) H \in F$. However $(U \# N)_{H}$ is isomorphic to a direct sum of $d$ copies of $U,(V \# N)_{H}$ is isomorphic to a direct sum of $d$ copies of $V$ and therefore since $F$ is $R_{0}$-closed (5) is proved.

(9) Let $H \in U(p, q)$ and let $V$ be a $z_{p} H$-module. Then $H \in F$ if and only if $V H \in F$.

For, there is a chain of submodules

$$
V=V_{0}>V_{1}>V_{2}>\ldots>V_{s}=0
$$

in which the factors $V_{i-1} / V_{i}(1 \leq i \leq s)$ are completely reducible. It follows from (5) by induction on $i$ that $\left(d^{i} V\right) H \in F$ if $V_{i} H \in F$ and that $\left(d^{i} V_{i}\right) H \in F$ if $V H \in F$. In particular when $i=s$ we have that (10) $H \in F \quad$ if $\quad V H \in F$,

which is one half of what we want, and also that $\left(\mathcal{d}^{S} V\right) H \in F$ if $H \in F$. 
Now

$$
\dot{d}^{s} V=\left(d^{s}-1\right) V \oplus V
$$

and so it follows from (10) that $\left(d^{S} V\right) H \in F$ implies $V H \in F$ (replacing $H$ by $V H$ and $V$ by $\left.\left(d^{5}-1\right) V\right)$. This completes the proof of (9).

Proof of Theorem. We use induction on the order of groups in $U(p, q)$. Let $G \in U(p, q)$ have a minimal normal subgroup $M$. We show that $G \in F$ if $H=G / M \in F$. The hypothesis $H \in F$ means that

$$
W=M w r H \dot{\epsilon} \text { : }
$$

this because of (9). Let $A$ be the base group of $W$. By the KrasnerKaloujnine embedding $G$ may be regarded as a subgroup of $W$ supplementing $A: W=A G$. There is a natural semi-direct product $X=A G$ from which there are two natural homomorphisms onto $W$ :

$$
(g, x) \rightarrow(g M) x \text { and }(g, x) \rightarrow g x, g \in G, x \in A,
$$

whose kernels intersect trivially. Consequently $X \in F$, if $H \in F$. A final application of (9) yields that $G \in F$ if $X \in F$. This completes the induction and the proof of the theorem.

\section{References}

[1] T.R. Berger, R.A. Bryce and John Cossey, "Quotient closed metanilpotent Fitting classes", J. Austral. Math. Soc. Ser A. 38 (1985), 157-163.

[2] R.A. Bryce and John Cossey, "Metanilpotent Fitting classes", J. Austral. Math. Soc. 17 (1974), 285-304.

[3] R.A. Bryce and John Cossey, "Subdirect product closed Fitting classes". Proc. Second Int. Conf. Theory of Groups, Australian National University, 1973.(Springer-Verlag, BerlinHeidelberg-New York, 1974).

[4] R.A. Bryce and John Cossey, "Subgroup closed Fitting classes are formations." Math. Proc. Cambridge Philos. Soc. 91 (1982), 225-258.

[5] Trevor O. Hawkes, "On Fitting formations", Math. 2. 117 (1970), 177-182. 
Department of Pure Mathematics,

Australian National University,

G.P.O. Box 4,

CANBERRA，

ACT 2601.

Australia. 